\title{
Diacronie
}

Studi di Storia Contemporanea

$N^{\circ} 16,4 \mid 2013$

Le monarchie nell'età dei nazionalismi

\section{Simboli e tradizioni "inventate" alla corte di Abdülhamid II}

\section{Federico Donelli}

\section{(2) OpenEdition}

\section{Journals}

\section{Edizione digitale}

URL: http://journals.openedition.org/diacronie/923

DOI: 10.4000/diacronie.923

ISSN: 2038-0925

\section{Editore}

Association culturelle Diacronie

\section{Notizia bibliografica digitale}

Federico Donelli, « Simboli e tradizioni "inventate" alla corte di Abdülhamid II », Diacronie [Online], № 16, 4 | 2013, documento 9, Messo online il 01 décembre 2013, consultato il 20 avril 2019. URL : http:// journals.openedition.org/diacronie/923 ; DOI : 10.4000/diacronie.923 


\section{Diacronie}

\section{9/}

\section{Simboli e tradizioni "inventate" alla corte di Abdülhamid II}

Federico DONELLI*

A seguito di una breve esperienza costituzionale, il sultano ottomano Abdülhamid II inaugurò una nuova fase di potere autoritario, legittimato e rafforzato da un'ideologia imperiale. La struttura di questa ricalcò i nazionalismi ufficiali promossi da altre dinastie europee, nella comune speranza di disinnescare le forze centrifughe interne ai rispettivi imperi. Parallelamente ad un pan-islamismo difensivo, si assistette al recupero del titolo di califfo ed alla ripresa di pratiche e simboli, gradualmente ritualizzati, divenuti ricorrenti nella vita quotidiana ottomana, con l'intento di evocare il potere e l'onnipresenza del sultano. Ad emergere è l'immagine di un impero per nulla passivo ed inerme di fronte alle sfide della modernità, nonché la figura di un sultano il cui operato, a lungo sottostimato dalla storiografia occidentale, ha saputo contraddistinguersi per capacità innovativa.

\section{Introduzione}

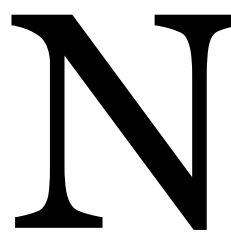

el corso del XIX secolo, al pari delle monarchie europee, il sultano ottomano avviò un processo di ridefinizione della propria autorità dinastica nel tentativo di rafforzarne la legittimità, sia nei confronti dei sudditi sia del mondo esterno, fornendo un nuovo, o quanto meno supplementare, fondamento "nazionale" alla propria istituzione. Un'ardua sfida per un grande impero multinazionale dove la minaccia di emergenti etno-nazionalismi obbligò a mobilitare i sudditi intorno a nuove linee identitarie nel tentativo di forgiare un nuovo tipo di appartenenza ottomana. Come i nazionalismi liberali anche le dinastie imperiali 
recuperarono dal passato pratiche e simboli, ritualizzandoli ed istituzionalizzandoli, nel tentativo di affermare la propria continuità con un passato storico accuratamente selezionato.

La rilevanza politica di Abdülhamid II è stata sottostimata dalla storiografia occidentale, a lungo influenzata negativamente dalla propaganda europea di inizio XX secolo volta a promuovere l'immagine di un tiranno reazionario assetato di sangue ${ }^{1}$. Solamente a partire dal 1960 è iniziata una revisione del suo operato attraverso nuove ricerche e analisi ${ }^{2}$. Il sultano, per quanto autoritario nei metodi di governo, non fu un conservatore, promuovendo riforme in chiave moderna conformi a quelle del periodo precedente (Tanzimat). Nonostante non nutrisse simpatie per il costituzionalismo né tanto meno per la democrazia, non sarebbe corretto considerarlo un retrogrado reazionario ${ }^{3}$, in quanto la sua azione fu costantemente orientata alla modernità. Abdülhamid II era un modernizzatore, non un occidentalista, che guardò all'Europa come modello da cui apprendere aspetti positivi.

Il lavoro, lungi dal tacere né tanto meno giustificare i metodi e le violenze perpetrate da Abdülhamid II, in particolare nei confronti delle comunità armene di cui molto si è scritto e si continua a scrivere 4 , vuole esporre la modernità politica dell'impostazione hamidiana, enfatizzando l'utilizzo di uno strumento di legittimazione tipico del periodo quali le "tradizioni inventate", combinazione di elementi religiosi e patriottici nonché creazione di simboli e pratiche socio culturali aggreganti. Utilizzando fonti di tipo secondario, l'articolo promuove un'interpretazione del periodo hamidiano da una prospettiva inedita: riprendendo quanto teorizzato da Hobsbawm5, è infatti possibile sostenere che le tradizioni inventate hamidiane abbiano avuto la finalità di rafforzare la coesione sociale e l'appartenenza ad una comunità oltre che legittimare un'istituzione e un sedimentato rapporto d'autorità in un contesto di rapido cambiamento.

\footnotetext{
${ }^{1}$ Influenza ben visibile nei primi lavori sul sultano: ROY, Gilles, Abdul-Hamid. Le sultan rouge, Paris, Payot, 1936; HASLIP, Joan, The Sultan. The life of Abdul Hamid II, New York, Holt, Rinehart and Winston, 1958.

${ }_{2}^{2}$ ZÜRCHER, Erik J., Storia della Turchia, Roma, Donzelli, 2007, p. 95.

3 MANSFIELD, Peter, A History of Middle East, New York, Penguin, 2003, p. 87.

4 Sull'argomento si veda: AKCAM, Taner, From Empire to Republic: Turkish nationalism and the Armenian Genocide, London, Zed Books, 2004; FLORES, Marcello, Il genocidio armeno, Bologna, Il Mulino, 2006; LEWY, Guenter, The Armenian Massacres in Ottoman Turkey: A Disputed Genocide, Glen Canyon, Utah University Press, 2007; GÖÇEK, Fatma Muge, NAIMARK, Norman M., SUNY, Ronald G. (a cura di), A Question of Genocide: Armenians and Turks at the End of the Ottoman Empire, Oxford/New York, Oxford University Press, 2011.

5 HOBSBAWM, Eric J., RANGER, Terence (a cura di), L'invenzione della tradizione, Torino, Einaudi Editore, 2002, pp. 11-12.
} 
Comprendere la svolta ideologica e identitaria promossa da Abdülhamid II, il suo carattere innovativo, significa riconoscere la lungimiranza politica di un sultano che più di ogni altro colse il senso di precarietà dell'impero e più in generale dell'intero universo islamico.

\section{Il particolare contesto socio-politico nei primi anni di regno}

Le radici della svolta hamidiana affondano nella firma degli accordi di Santo Stefano (3 marzo 1878) con l'Impero Russo, evento che segnò uno spartiacque politico, culturale e psicologico della storia ottomana ${ }^{6}$. La logica degli equilibri, alla base del Congresso di Berlino (giugno-luglio 1878), portò alla stipula di un nuovo trattato in cui l'abile utilizzo dell'intrigo politico e dell'arte diplomatica da parte dei delegati ottomani divise le posizioni europee attenuando le perdite territoriali7. Ciononostante, la carta geografica dell'intera regione venne ridisegnata introducendo l'elemento etniconazionale tra popolazioni fino a quel momento organizzate in comunità confessionali, istituzionalizzate nel sistema ottomano dei mille $^{8}$. Il modello di nazionalismo etnico, in particolare quello territoriale e ortodosso promosso dallo Zar, oltre a destabilizzare i territori balcanici rafforzò, per reazione, la coscienza identitaria musulmana ${ }^{9}$. Inoltre, la consapevolezza di essere soggetto passivo del concerto europeo accentuò un senso di frustrazione e rabbia latente, elemento risultato determinante alla svolta pan-islamica del sultano.

Il ridimensionamento territoriale ebbe conseguenze devastanti per l'impero che perse circa un terzo dei propri domini e più del 20\% della propria popolazione, accentuando l'immigrazione forzata dei musulmani che, a partire dalla sconfitta nella guerra russo-ottomana del $1774^{10}$, aveva assunto proporzioni significative ${ }^{11}$. L'esodo di

\footnotetext{
${ }^{6}$ KARPAT, Kemal H., The Politicization of Islam: Reconstructing Identity, State, Faith, and Community in the Late Ottoman State, Oxford/New York, Oxford University Press, 2002.

7 MANSFIELD, Peter, op. cit., p. 92.

8 KARPAT, Kemal, Millets and Nationality: The Roots of the Incongruity of Nation and State in the Post Ottoman Era, in BRAUDE, Benjamin, LEWIS, Bernard (eds.), Christians and Jews in the Ottoman Empire, vol. 1, The Functioning of a Plural Society, New York-London, Holmes \& Meir, 1980, pp. 141-170.

9 DEL ZANNA, Giorgio, I cristiani e il Medio Oriente (1798-1924), Bologna, Il Mulino, pp. 177178.

${ }^{10} \mathrm{Fu}$ la Quinta guerra russo-ottomana (1768-1774) conclusasi con la perdita per la prima volta di territori abitati da popolazioni musulmane.

${ }^{11} \mathrm{Nel}$ giro di un secolo l'avanzata russa coinvolse le popolazioni musulmane di diverse aree geografiche dell'impero dalle coste del Mar Nero (500.00o) alle regioni caucasiche ad est (1,2 milioni) e, infine, le province balcaniche (circa 1,5 milioni). McCARTHY, Justin, Death and Exile: The Ethnic Cleansing of Ottoman Muslims (1821-1922), Princeton, Darwin, 1995.
} 
migranti musulmani, considerati dalle autorità ottomane muhaciri2, mutò la composizione dell'impero e la popolazione di fede islamica passò dal ( 68\% ) al ( 76\% ) con i turchi componente etnica maggioritaria ${ }^{13}$.

A risentire di questi stravolgimenti furono soprattutto le campagne, dove si diffuse rapidamente un movimento di revival islamico, una corrente proveniente dal basso, completamente diversa da quella promossa dai Giovani Ottomani. Se l'intellighenzia ottomana aveva proposto un'idea sorta dal contatto con la moderna cultura europea nei suoi più disparati campi - scienza, filosofia, politica, letteratura - il movimento "revivalista" aveva un carattere popolare in cui il rigore islamico si combinava alle radici mistiche delle confraternite sufi $^{14}$. Puntando il dito contro la modernità, identificata nelle potenze europee e, di riflesso, nei millet cristiani, il movimento fu un contenitore del malessere e delle frustrazioni della popolazione musulmana che ambiva al ripristino del giusto ordine islamico, in cui i non musulmani (dhimmi) sono considerati soggetti protetti e tollerati ma di status inferiore ai musulmani. Nonostante il movimento revivalista sia rimasto un fenomeno del tutto distinto dalle successive correnti pan-islamiche ${ }^{15}$, ebbe il merito di infondere una nuova consapevolezza nelle masse, persuadendo Abdülhamid II del fatto che l'Islam restasse il vero collante in grado di tenere unita la popolazione ottomana.

Nei primi anni di regno Abdülhamid II consolidò il processo di centralizzazione e modernizzazione amministrativa avviato un secolo prima da Selim III (1789-1807) e Mahmud II (1808-1839). Ad agevolare tali sviluppi fu lo straordinario incremento dei mezzi di comunicazione: dal telegrafo alla ferrovia passando per i battelli a vapore ${ }^{16}$. Le nuove reti, oltre a favorire l'economia ottomana ${ }^{17}$, rappresentarono strumenti di potere tipici dello Stato moderno, quale l'impero ambiva ad essere, collegando il centro alle periferie ed esercitando per la prima volta una presa costante su tutti i sudditi. Le prime iniziative politiche di Abdülhamid II, quindi, non segnarono una rottura con il passato ma piuttosto una continuità assicurata dalla presenza di funzionari vicini alle

12 I migranti vennero presentati dalle autorità ottomane come muhacir, ossia fedeli che al pari del Profeta costretto a fuggire da Mecca a Medina, erano costretti ad abbandonare la propria casa per conservare la fedeltà all'Islam.

13 DEL ZANNA, Giorgio, op. cit., p. 181.

14 Fondamentale risultò essere l'influenza della confraternita Nakșibendi, che guadagnerà ulteriore prestigio e visibilità in questa convulsa fase garantendosi il futuro appoggio dello stesso sultano Abdülhamid.

15 Il movimento non concepì mai una forma di unità politica islamica sotto la guida del potere califfale. KARPAT, Kemal H., op. cit., pp. 44-47; DEL ZANNA, Giorgio, op. cit., p. 171.

${ }_{16}$ ZÜRCHER, Erik J., op. cit., pp. 94-95.

${ }_{17}$ Le linee ferroviarie collegavano i porti alle zone produttive interne del Paese, mentre i battelli collegavano in maniera sempre più rapida i porti ottomani a quelli europei. Il loro sviluppò segnò il definitivo ingresso dell'economia ottomana nel sistema capitalistico. 
idee dei Giovani Ottomani, tra cui il primo gran vizir Mehmed Said Paşa (1830-1914) che promosse l'ennesima riforma dell'istruzione nella speranza di garantire la formazione di nuovi ranghi burocratico amministrativi. L'alto grado di alfabetizzazione raggiunto all'inizio del XX secolo, nonostante tassi di molto inferiori agli standard europei del periodo, mostra la continuità con i propositi delle Tanzimat e rappresenta un grande successo di Abdülhamid II ${ }^{18}$.

A determinare il cambio di atteggiamento da parte del sultano furono la conquista francese della Tunisia (1881) e quella britannica dell'Egitto (1882) che accentuarono tra la popolazione la sensazione di isolamento e accerchiamento, convincendo i vertici del potere che l'ottomanismo impostato da Alì Paşa e Fuad Paşa, inteso come unità di diverse etnie in un'unica cittadinanza (Ittihad-i Anasir), avesse fallito. Infatti, l'ottomanismo delle Tanzimat - cittadinanza ottomana laica ed egualitaria (in termini religiosi non sociali) come risposta ai particolarismi etnico-confessionali - ottenne inizialmente un buon supporto ma, in breve tempo, risultò essere più forza disgregante che unificante ${ }^{19}$.

Gli sviluppi internazionali accelerarono un processo innescatosi da tempo. Tra il 1878 e il 1880, quando le riforme tardavano a dare risultati tangibili e le forze centrifughe dell'impero avevano aumentato le proprie pressioni acuendo il malessere e la mortificazione delle masse musulmane, i timori del sultano di possibili complotti interni, reminiscenza di quanto accaduto al suo predecessore, assunsero la forma di vera e propria ossessione, spingendolo ad inasprire il carattere autoritario del governo. Il delirio progressivo di Abdülhamid II portò alla costituzione di un fitto sistema di controllo su tutte le componenti della società, con il diffondersi della figura del hafiye, informatore-spia, e della censura ${ }^{20}$.

${ }_{18}$ Tra il 1867 e il 1895 sia il numero degli studenti sia quello delle scuole era più che raddoppiato. SOMEL, Selcuk Aksin, The Modernization of Public Education in the Ottoman Empire, 1839-1908. Islamization, Autocracy, and Discipline, Leiden, Brill, 2001.

19 BRAUDE, Benjamin, LEWIS, Bernard (eds.), op cit., pp. 30-31.

${ }^{20}$ GEORGEON, François, Abdülhamid II. Le Sultan-Calife (1876-19o8), Paris, Fayard, 2003, pp. 160-175. 


\section{La svolta hamidiana, promozione dell'ottomanismo pan-islamico ${ }^{21}$}

La svolta islamista del regime di Abdülhamid II fu parallela a quella autoritaria. Nel giro di poco tempo il sultano non riconvocò il Parlamento, giudicando il popolo ottomano immaturo per godere di un regime costituzionale, e rafforzò il proprio potere esautorando i membri della vecchia élite burocratica a favore di funzionari a lui totalmente sottomessi. I principali obiettivi di Abdülhamid II erano di riacquisire il controllo interno, estromettendo gli uomini della Porta e arrestare il declino dell'impero. Valutati questi due obiettivi, per Abdülhamid II la svolta islamica non fu dettata da convinzioni religiose quanto invece considerata in maniera pragmatica un mezzo per raggiungere i propri fini.

Il sultano, sebbene si richiami all'idea di un'unità ottomano-islamica (Ittihad-i Islam) dei Giovani Ottomani, decise di cambiare l'impostazione ideologica dell'ottomanismo liberale facendo dell'elemento islamico il nucleo di una nuova ideologia imperiale. Forte dell'appoggio delle masse, si convinse che la religione potesse essere una risorsa determinante al rinnovamento e alla sopravvivenza dell'impero ${ }^{22}$. Alla base del cambiamento ideologico operato da Abdülhamid II si celava la ferma contrarietà alle correnti ideologiche europee (liberalismo, nazionalismo, costituzionalismo) considerate forze distruttive nonché veicoli dell’imperialismo ${ }^{23}$.

La modernità del discorso affiora dalla concezione stessa di Islam del sultano. Per Abdülhamid II, infatti, l'Islam era una religione dinamica pronta ad aprirsi ai progressi delle scienze occidentali, idea, questa, radicata nell'eterodosso Islam turco ${ }^{24}$. Al pari dei Giovani Ottomani anche il sultano riconobbe come l'Occidente fosse più avanzato dal punto di vista tecnologico e, pertanto, toccasse all'impero adottare diverse abilità e

\footnotetext{
${ }^{21}$ Il termine panislamismo comparve in Europa nella seconda metà del XIX secolo con l'intento di indicare il ritorno in chiave identitaria del sentimento religioso tra i musulmani. Di pan-Islam si parlò apertamente per la prima volta nel $\mathbf{1 8 8 0}$, in un articolo del giornalista francese Gabriel Charmes, nel descrivere le resistenze tunisine all'occupazione francese. Si veda: LANDAU, Jacob M., The politics of pan-Islam, Oxford, Clarendon Press, 1990, pp. 2-6.

${ }^{22}$ DEL ZANNA, Giorgio, op. cit., p. 187.

23 DEMIRAG, Yelda, «Pan-Ideologies in the Ottoman Empire against the West: From PanOttomanism to Pan-Turkism», in The Turkish Yearbook of International Relations, 36, 2005, pp. 139-156, si vedano in particolare le pp. 147-148. Sull'argomento si veda anche: KHALID, Adeeb, Pan-Islamism in Practice: The Rhetoric of Muslim Unity and its Uses, in ÖZDALGA, Elizabeth (ed.), Late Ottoman Society: The Intellectual Legacy, New York, Routledge, 2010, pp. 201-224.

24 DEL ZANNA, Giorgio, op. cit., p. 191.
} 
conoscenze occidentali per riuscire a colmare il gap tra le due realtà, evitando però la semplice emulazione ${ }^{25}$.

Il sultano sostenne una forma di Islam in cui elementi dell'ortodossia combinavano quelli delle confraternite che durante il suo regno acquisirono credito a palazzo; in particolare la Nakşibendi ${ }^{26}$ risultò funzionale ai progetti del sultano grazie alla capacità di mobilitare le masse contro la penetrazione culturale ed economica straniera ${ }^{27}$. Le confraternite sufi contribuirono alla costruzione di un'identità basata sulla solidarietà religiosa, attraverso una fitta rete di legami sociali interpersonali volti al sostegno reciproco ${ }^{28}$. Oltre agli sheikh sufi, tra cui un giovane curdo Said Nursî29, il sultano si servì dell'apporto di alcuni tra i più illustri musulmani del periodo tra i quali spicca la figura di Jamal ad-Din al-Afghani (1838-1887) la cui presenza a Costantinopoli servì ad aumentare il prestigio della corte ${ }^{30}$.

Abdülhamid II ebbe la lungimiranza politica di comprendere la necessità di appellarsi alla solidarietà musulmana dei sudditi promuovendo un'identità sociopolitica islamica, trascurata dai suoi predecessori, in modo da rinsaldare la popolazione intorno alla figura del sovrano. Il comune vincolo religioso avrebbe dovuto riflettere i nuovi equilibri demografici dell'impero e rappresentare un fattore di unità in grado di porsi al di sopra dei molteplici particolarismi nazionali. L'idea era che dall'Islam le élite ottomane avrebbero ricavato la base emotiva per mobilitare le masse, solo l'Islam avrebbe fornito i simboli in grado di competere con la mobilitazione nazionale ${ }^{31}$; quindi, l'Islam divenne veicolo di consenso.

L'elaborazione pan-islamica di Abdülhamid II risultò essere un’ideologia politicoreligiosa non alternativa all'adattamento alla modernità, ma condizionava tale sviluppo al mantenimento del proprio peculiare carattere islamico ottomano. L’identità imperiale hamidiana restituì un ruolo determinante all'Islam, considerandolo

25 KARPAT, Kemal H., op. cit., pp. 168-170.

26 Diversi sheikh Nakşibendi si resero persino protagonisti della guerra russo-ottomana combattendo in prima linea e svolgendo un importante opera di propaganda tra le popolazioni dei villaggi. DERINGIL, Selim, The Well-Protected Domains: Ideology and the Legitimation of Power in the Ottoman Empire 1876-1909, London, I. B. Tauris, 1999, p. 63.

${ }_{27}$ YAVUZ, Hakan M., Islamic Political Identity in Turkey, New York, Oxford University Press, 2003 , p. 138.

28 Tale meccanismo, con i dovuti limiti, risulta non molto diverso da quello innescato dalle stesse confraternite e dai movimenti di Islam popolare nella Turchia contemporanea a partire da metà anni Ottanta.

${ }^{29}$ Said Nursî fondò il movimento mistico nur (in turco Nurculuk) ad inizio del XX secolo. Un movimento che si sviluppo a seguito della censura kemalista riemergendo nella seconda metà del secolo come uno dei principali movimenti socio religiosi turchi. Oggi la principale branca è quella fondata e guidata da Fethullah Gülen.

3o BERKES, Niyazi, The Development of Secularism in Turkey, Montreal, McGill University Press, 1998, pp. 268-270.

${ }^{31}$ DERINGIL, Selim, op. cit., p. 18. 
l'elemento culturale primario32; combinando la tradizione a graduali aperture verso la modernità creò un quadro di valori e principi di riferimento per le forze sociali più dinamiche che avrebbero dovuto, nei piani del sultano, trainare la ripresa e il rinnovamento di tutto l'impero33.

Centrale in questo processo di creazione di un senso di appartenenza ottomana fondato sulla lealtà alla dinastia e sulla comune fede islamica risultò una generale mobilitazione simbolica centrata sul recupero dell'immagine di califfo.

\section{L’immagine di "califfo beato", richiamo agli Zar di Russia}

La scelta del sultano di recuperare il ruolo di califfo per rilegittimare la propria autorità, risultò simile a quella degli altri due grandi imperi multietnici, Asburgico e Russo, che avevano da poco promosso il "nazionalismo ufficiale", ossia il richiamo di motivi e valori peculiari del nazionalismo all'interno dell'ideologia dinastica ${ }^{34}$.

Abdülhamid II riprese elementi e pratiche radicati nella cultura e nell'identità ottomana ammantandoli di norme e valori nuovi, moderni, con l'intento di rinsaldare i vincoli imperiali; centrale fu il recupero, la ricostruzione e, in molti casi l'invenzione, di una complessa simbologia, combinazione di tradizione e modernità, per consolidare il sentimento di appartenenza e lealtà al sultano. L'enfasi posta sulla veste di califfo fu icona di una nuova identificazione etnico-religiosa rispecchiando quelle che, secondo Hobsbawm, rappresentano «le icone più soddisfacenti da un punto di vista protonazionale» perché «specificamente associate a un re o un imperatore imbevuto di carattere divino, il cui regno coincide con la futura nazione» 35.

Il meccanismo risultò comune a quello di altre dinastie legittimiste, più specificatamente quella dei Romanov, dove gli Zar, a partire da Nicola I, cercarono di creare un legame diretto con i sudditi sfruttando una sintesi tra i tradizionali miti russi e la fede ortodossa, generando un nazionalismo, per l'appunto "ufficiale”, fondato sulla

\footnotetext{
${ }^{2}$ KIESER, Hans-Lukas, Turkey Beyond Nationalism: Towards Post-Nationalist Identities, London, I. B. Tauris, 2006, p. 7

33 KARPAT, Kemal H., op. cit., p. 115.

34 SETON-WATSON, Hugh, Nations and States: An Enquiry Into the Origins of Nations and the Politics of Nationalism, London, Taylor \& Francis, 1977, p. 148. Si veda anche: ROSHWALD, Aviel, Ethnic Nationalism and the Fall of Empires: Central Europe, the Middle East and Russia (1914-23), London, Routledge, 2003, pp. 49-63.

35 HOBSBAWM, Eric J., Nations and Nationalism since 1780, Cambridge, Cambridge University Press, 1990, pp. 72-73 [corsivo mio].
} 
formula autocrazia, ortodossia e nazionalità ${ }^{36}$. Formula che all'interno dell'Impero Ottomano si tradusse in: Dio, il Profeta e il Sultano37. Come Nicola I e Alessandro III alimentarono l'immagine di "Zar beato", Abdülhamid II reintrodusse il titolo di califfo in quanto "personaggio sacro", nella speranza di plasmare un legame di sacralità diretto tra popolo e sovrano alternativo a quello proposto dalle forze "eversive" dei nazionalismi 38 .

L'utilizzo politico del califfato non fu una novità, già in passato venne sfruttato durante le guerre con altre entità musulmane, come contro l'Impero Safavide sciita (XVI secolo) o, nel tardo XVIII secolo, in risposta al diffondersi della minaccia wahhabita nell'Hijaz ${ }^{39}$. Inoltre ad inizio XIX secolo, il califfato diventò strumento di contrasto alla penetrazione europea, consentendo alla Porta di rivendicare il proprio diritto di tutela delle comunità musulmane poste sotto dominio della cristianità al pari di quanto fatto dalle potenze europee con le comunità cristiane dell'impero40. A differenza dei precedenti, il richiamo califfale di Abdülhamid II rappresentò soprattutto l'occasione di presentare sotto diversa veste la propria sovranità. Il titolo di califfo - in ottica hamidiana - avrebbe garantito agli occhi dei musulmani un sovrano giusto, virtuoso, semplice e generoso le cui azioni sarebbero state conformi alla morale islamica (ahlak)41. Una completa innovazione aderendo però a modelli tradizionali attraverso la costruzione di un rapporto di continuità in larga misura illusorio ${ }^{42}$.

L'attribuzione del titolo di califfo aveva da sempre avuto bisogno della legittimazione giuridica assicurata dalla scuola religiosa hanafita considerata la "setta ufficiale" (mezheb-i resmiye) della dinastia. La preferenza ottomana per la scuola hanafita deriva proprio dall'interpretazione flessibile data alla veste di califfo; secondo questa, infatti, un sovrano forte e capace, indipendentemente dall'appartenenza al clan del Profeta, quello dei Quraysh, deve essere ritenuto califfo legittimo di tutti i musulmani a condizione che protegga l'Islam e sostenga la Şeriat (shari’a). L'assenza di discendenza ottomana dai Quraysh richiese un'ampia propaganda soprattutto nelle

36 CHAPMAN, Tim, Imperial Russia (1801-1905), London, Routledge, 2001, pp. 55-56; RIASANOVSKY, Nicholas Valentine, Nicholas I and Official Nationality in Russia: 1825-1855, Berkeley-London, University of California Press, 1959, pp. 74-78.

37 KARPAT, Kemal H, Ottoman Past and Today's Turkey, Leiden-Boston, Brill, 200o, p. 53.

38 DERINGIL, Selim, op. cit., p. 17.

39 FINKEL, Caroline, Osman's Dream: The History of the Ottoman Empire, New York, Basic Books, 2005, p. 412.

$4^{\circ}$ BRAUDE, Benjamin, LEWIS, Bernard (eds.), op. cit., p. 31.

${ }^{41}$ GEORGEON, François, op. cit., pp. 198-199.

${ }^{42}$ HOBSBAWM, Eric J., RANGER, Terence (a cura di), op. cit., p. 11. 
province arabe ${ }^{43}$, dove attraverso la cooptazione di sheikh e notabili, riuscì a trasmettere ai fedeli arabi la nuova immagine di un sovrano pio, difensore dell'Islam 44 .

Le politiche hamidiane e l'immagine paternalista del sovrano, pur con esiti parziali, risultarono le prime forme pratiche di mobilitazione delle masse ottomane. Nessuna partecipazione attiva ma semplice consenso passivo delle masse, attraverso un graduale coinvolgimento a cerimoniali, festeggiamenti e pubbliche manifestazioni, in quanto espressione di appartenenza all'impero e di fedeltà verso il sultano45.

\section{L'invenzione delle tradizioni, sottile equilibro con la modernità}

Non bastando la legittimazione religiosa, il regime dovette iniziare a legittimare il proprio potere agli occhi della gente attraverso lo sfruttamento di dispositivi politici e simbolici; vennero recuperati e alimentati leggende e miti affinché imprimessero nelle menti dei sudditi l'idea di consenso e continuità ottomana con i passati califfati islamici. Molti di questi furono invenzioni del periodo precedente (XVIII secolo) riprese e strumentalizzate, come l'episodio del formale passaggio di consegne tra l'ultimo califfo Abbaside al-Mutawakkil III e il sultano Selim I, attraverso il dono del mantello califfale dopo la conquista dell'Hijaz (1516) ${ }^{46}$.

In poco tempo l'intera iconografia intorno alla figura del sultano assunse un carattere sacrale, supportato dall'invenzione della tradizione, che nel contesto ottomano risultò un'attenta rielaborazione di tradizioni a lungo taciute e ignorate, rivestite di significato regio attraverso il richiamo alle pratiche delle moderne corti europee ${ }^{47}$. Il simbolismo rivestì il regno in molteplici aspetti della vita del sultano e dei sudditi: dal vestiario alla nomenclatura usata in occasione di atti ed eventi pubblici fino

43 KAYALI, Hasan, Arabs and Young Turks: Ottomanism, Arabism, and Islamism in the Ottoman Empire (1908-1918), Berkeley, University of California Press, 1997, p. 36.

44 BÜSSOW, Johann, Hamidian Palestine: Politics and Society in the District of Jerusalem 1872-19o8, Leiden, Brill, 2011, pp. 392-395.

45 ÇETINKAYA, Doğan, On the Social origins of Turkish Nationalism: the anti-Greek movement in the Ottoman Empire (1910-1914), in LYBERATOS, Andreas, Social Transformation and Mass mobilization in the Balkan and Eastern Mediterranean Cities (1900-1923), Heraklion, Crete University Press, 2013, pp. 230-243.

${ }^{46} \mathrm{Si}$ ritiene sia stato appositamente creato per rafforzare la dinastia ottomana in un periodo di particolare crisi coinciso a cavallo del XVIII e XIX secolo. INALCIK, Halil, QUATAERT, Donald (eds.), An economic and social history of the Ottoman Empire, 130o-1914, Cambridge, Cambridge University Press, 1994, pp. 20-21. Studi sul XVI-XVII secolo ottomano dimostrano come la legittimità del potere risiedesse nelle vittorie militari e nell'efficienza delle politiche di governo piuttosto che su un richiamo ideologico trascendentale all'eredità del califfato. FLEISCHER, Cornell H., Bureaucrat and intellectual in the Ottoman Empire: the historian Mustafa Ali (1541-16oo), Princeton, Princeton University Press, 1986, p. 276.

47 HANIOĞLU, Şükrü, Modern Ottoman period, in HEPER, Metin, SAYARI, Sabri (ed.), The Routledge Handbook of Modern Turkey, New York, Routledge, 2012, p. 22. 
alle decorazioni e ai cerimoniali di palazzo48. I fastosi cerimoniali oltre a colpire gli ospiti, rappresentarono, come in altri contesti49, l'espressione di continuità storica e immutabilità in un periodo di crisi e vulnerabilità per la dinastia. L'elemento di novità del cerimoniale rispetto alla tradizione, introdotto anche dalle monarchie dei Romanov e degli Asburgo, era la misura in cui questo si rivolgeva ad un pubblico sempre maggiore determinando un primo ampio processo di edificazione delle masse ${ }^{5}$. Centrale era l'immagine del monarca, nello splendore dei suoi rituali, rivestita di un'aurea mitica alimentata dal progressivo ritiro dietro le mura del palazzo. Tale atteggiamento rappresentò una rottura rispetto a quello dei predecessori (Mahmud II e Abdülmecit) intenti ad alimentare l'immagine di moderno monarca pubblico pronto a mostrarsi tra la gente per dare manifestazione diretta del potere dello Stato.

Al contrario, la figura di Abdülhamid II venne gestita attraverso un sistema di simboli ricorrenti nella vita quotidiana ottomana che, sostituendosi alle sue apparizioni pubbliche, avevano l'intento di evocare il suo potere e la sua onnipresenza. Questo processo di ridimensionamento dell'immagine pubblica, dettato dall'ossessione per la propria sicurezza, agevolò l'introduzione di un nuovo modo di relazionarsi con i sudditi, attraverso l'utilizzo di una sintassi simbolica basata quasi interamente su motivi islamici. Abdülhamid II sfruttò un sistema di legittimazione tipico della modernità, incentrato sulla figura paternalistica del sovrano, ammantandola però di una veste islamica.

La pratica più ripetitiva e importante nella vita dei musulmani, ossia la preghiera, in particolare quella del venerdì alla presenza del sultano, fu interessata dal processo di ricostruzione simbolico identitario hamidiano e assunse una teatralità volta ad impressionare i fedeli piuttosto che a soddisfarne i bisogni spirituali. Abdülhamid II, seguendo l'esempio delle corti europee, ornò la preghiera del venerdì di un protocollo nuovo in cui a risaltare fosse la figura stessa del sovrano non solo allinterno della moschea ma anche prima e dopo la funzione. La preghiera rientra in quelle definite da Hobsbawm come consuetudini51; la "tradizione inventata", invece, era rappresentata dall'insieme degli aspetti formali e delle pratiche ritualizzate che circondavano l'azione sostanziale. La cerimonia esibiva una significativa mescolanza di vecchio e nuovo,

${ }^{48}$ DERINGIL, Selim, op. cit., pp. 15-43.

49 Si veda l'analisi dell'evolversi del cerimoniale della monarchia britannica ottocentesca: CANNADINE, David, Il contesto, la rappresentazione e il significato del rito: la monarchia britannica e linvenzione della tradizione, in HOBSBAWM, Eric J., RANGER, Terence (a cura di), op. cit, pp. 99-156.

$5^{\circ}$ HOBSBAWM, Eric J., RANGER, Terence (a cura di), op. cit., p. 271.

${ }^{51}$ HOBSBAWM, Eric J., RANGER, Terence (a cura di), op. cit., pp. 4-5. 
tradizioni musulmane e usi occidentali, divenendo occasione di incontro tra le pratiche islamiche e il rigido protocollo europeo52.

La costante ricerca di sottili equilibri tra tradizione e modernità interessarono non solo il cerimoniale ma anche il sistema araldico dell'impero, su tutti lo stemma della dinastia di Osman (Arma-i Osmani) simbolo dello Stato ottomano che, nonostante fosse in uso dai tempi di Mahmud II (1808-1839), venne uniformato per la prima volta da Abdülhamid II. Lo stemma rifletteva il percorso attraversato dall'impero negli ultimi decenni: al suo interno erano distinguibili una serie di elementi in perfetto equilibrio tra loro richiamanti la tradizione turca ottomana islamica ed altri assimilabili al progresso laico occidentale. Il tema centrale dell'emblema ruotava intorno alla continuità tra passato e presente, tradizione e modernità ben enfatizzate dalla presenza di due voluminosi tomi, uno raffigurante la legge islamica Şeriat e l'altro i codici laici europei53.

Il rischio principale del processo hamidiano era che la modernità potesse scontrarsi con l'autenticità islamica; spettava così allo stesso sultano ed ai suoi consiglieri giungere a compromessi. Venne così abolita la pratica di esporre i ritratti del sultano nei luoghi pubblici, inaugurata durante le Tanzimat con l'obiettivo di far apparire il sovrano come un "pubblico monarca" e, in una manifestazione iconoclasta dell'Islam più rigido, sostituita da drappi ricamati recanti la scritta "Lunga Vita al Sultano" 54.

Il ripristino dell'elemento islamico nell'ideologia politica ufficiale comportò che lo Stato dovesse assicurarsi il monopolio della sacralità, reso possibile attraverso il minuzioso controllo della stampa e dell'importazione di copie del Corano. I timori erano soprattutto rivolti alla provenienza di testi dalla Persia, quindi sciiti, ma anche sulle copie stampate in Egitto sotto la supervisione dell'Università al-Azhar, perché c'era il sospetto di eventuali calunnie e mistificazioni di penetrazione europea. Venne per questo istituita un'apposita commissione per l'Ispezione dei Corani che dimostra quanto la Porta vivesse la situazione come una minaccia reale per la propria legittimità, alimentando un'idiosincrasia figlia della psicologia di Abdülhamid II che trovò riscontro nei progetti europei per l'istituzione di un califfato arabo. L'aspetto paradossale fu che l'ossessione della Porta si concentrò sui testi sacri ignorando invece pubblicazioni che sarebbero risultate anche più pericolose per la stabilità del regno, come i lavori di Darwin, Spencer e Mill, venduti liberamente nei negozi di

\footnotetext{
${ }^{2}$ Ibidem, pp. 22-25.

53 DERINGIL, Selim, op. cit., p. 27.

54 FINDLEY, Carter V., Ottoman Civil Officialdom: A Social History, Princeton, Princeton University Press, 1989, p. 135.
} 
Costantinopoli e diventati base della formazione positivista dei futuri membri del CUP (Comitato Unione e Progresso)55.

Strumento migliore per inculcare il legame tra sacralità e figura del monarca era rappresentato dallo sfruttamento di uno strumento tradizionale dello Stato moderno, ossia l'istruzione di massa. Sull'esempio di quanto avvenuto pochi decenni prima nell'Impero Russo su disegno del Conte Sergei Uvarov, Ministro dell'educazione nonché responsabile della "russificazione", l'Impero Ottomano innescò un processo di standardizzazione dell'istruzione ridando centralità all'istruzione religiosa56. Queste politiche risultarono un utile strumento per integrare le molte realtà tribali presenti nell'impero favorendo il passaggio da nomadi a "ottomani"; in particolare questo fu il caso di molti gruppi curdi dell'Anatolia orientale, un processo non molto diverso da quello avvenuto precedentemente in Francia quando Parigi concentrò i propri sforzi nel trasformare i contadini in "francesi" 57 . L'altro strumento utilizzato fu la costituzione di speciali corpi militari, truppe di cavalleria curda (Hamidiye), create sul modello cosacco e protagoniste dei massacri armeni tra il 1894 e il $1897^{8}$.

Molta dell'impostazione politica hamidiana fu volta ad accentuare il legame e il controllo sulle province arabe, il cui sistema amministrativo tradizionale le rendeva diverse dal resto dell'impero; in particolare il governo ottomano, attraverso l'uso equilibrato di elementi moderni e islamici, riuscì ad instaurare un collegamento con le classi urbane arabe in ascesa ${ }^{59}$. Se il compito di stimolare la lealtà verso il califfo venne delegato agli ulama, quello di fare da collegamento tra Stato centrale e province fu affidato alle famiglie di proprietari terrieri che mandavano i loro figli a Costantinopoli per svolgere funzioni all'interno della burocrazia e dell'amministrazione ${ }^{60}$.

Nelle province arabe come altrove emerse lo sfruttamento da parte del governo dell'architettura per manifestare le presenza dello Stato in zone lontane, metodo esplicito per imporre una nuova interpretazione della storia e dell'appartenenza comune. Più importante e famosa fu la ferrovia dell'Hijaz costruita grazie alle offerte provenienti dalle comunità musulmane di tutto il mondo; pratica comune diventò

55 DERINGIL, Selim, op. cit., pp. 53-54.

${ }^{6}$ REID, James J., Crisis of the Ottoman Empire: Prelude to Collapse (1839-1878), Stuttgart, Steiner, 2000, pp. 380-382. Sull'argomento si veda anche: MILLER, Alexei, The Romanov Empire and Nationalism. Essays in the Methodology of Historical Research, Budapest-New York, Central European University Press, 2008, pp. 162-175.

57 DERINGIL, Selim, op. cit., p. 67. Il riferimento di Deringil è al testo: WEBER, Eugene, Peasants into Frenchmen: The Modernization of Rural France 1870-1914, Stanford, Stanford University Press, 1976.

${ }^{8}$ GEORGEON, François, op. cit., pp. 267-270.

59 KAYALI, Hasan, op. cit., p. 36.

${ }^{60} \mathrm{ABU}-\mathrm{MANNEH}$, «Sultan Abdulhamid II and Shaikh Abulhuda Al-Sayyadi», in Middle Eastern Studies, 15, 2/1979, pp. 131-153; si vedano in particolare le pp. 140-142. 
anche la costruzione di moschee (tradizione) e delle torri dell'orologio (modernità) che iniziarono a scandire il ritmo e il tempo della vita ottomana andando a sostituire le grida del muezzin ${ }^{61}$. Le ricorrenze o i riti inventati, quali compleanni e giubilei, erano occasione nell'impero come per altri Stati europei62, per richiamare un'ampia partecipazione popolare con l'inaugurazione di opere monumentali come l'acquedotto palestinese in occasione del sessantesimo compleanno di Abdülhamid II nel $1902^{63}$.

Il narcisismo del sultano lo spinse inoltre a coltivare la propria immagine al pari di quella dell'impero in campo internazionale attraverso lo sfruttamento dei mezzi più efficaci del periodo; la cosiddetta politica dell'immagine ${ }^{64}$ promosse la partecipazione dell'Impero Ottomano ai grandi eventi internazionali come le fiere e le esposizioni universali (Chicago 1891), ma anche l'utilizzo della fotografia come strumento di propaganda65. Gli sforzi per riabilitare l'immagine internazionale dell'impero dimostrano come il regno hamidiano non rifuggisse la modernità ma cercasse una via di progresso in grado di mediare con il proprio carattere storico ottomano islamico.

\section{Conclusione}

Valutare l'operato di Abdülhamid II e il contributo dato allo sviluppo in chiave moderna dello Stato ottomano risulta difficile perché tale valutazione non può esimersi da considerare i metodi autoritari di governo e le violenze perpetrate nei confronti della minoranza armena. Tuttavia, come sottolineato da Zürcher, è possibile notare che, di fronte all'aggressione del liberalismo e del nazionalismo, Abdülhamid II abbia reagito nella stessa maniera in cui reagirono altri due imperatori, Francesco Giuseppe d'Austria e lo Zar Alessandro III, proiettando verso la massa l'immagine paternalistica del sovrano e sottolineandone la sacralità e l'onnipresenza. La grande differenza, secondo lo stesso autore, derivò dall'utilizzo da parte di Abdülhamid II della religione come amalgama per la popolazione, una scelta che allontanò la lealtà nei suoi confronti da parte di due componenti fondamentali dell'impero: l'élite burocratico militare e la

\footnotetext{
${ }^{61}$ HANSSEN, Jens, Fin de Siècle Beirut: The Making of an Ottoman Provincial Capital, Oxford, Oxford University Press, 2005, p. 247.

62 HOBSBAWM, Eric J., RANGER, Terence (eds.), op. cit., pp. 260-262.

63 BÜSSOW, Johann, op. cit., p. 497.

64 GEORGEON, François, op. cit., p. 275.

${ }^{65}$ Sulle fiere internazionali si veda: DERINGIL, Selim, op. cit., pp. 153-164. Sulla fotografia si veda: GAVIN, Carney E. S. (ed.), Journal of Turkish Studies : Imperial Self-Portrait: The Ottoman Empire as Revealed in Sultan Abdul-Hamid II's Photographic Albums, 12, 1988. cit. in REID, James J., op. cit., p. 382.
} 
minoranza cristiana da cui dipendeva l'economia ottomana ${ }^{66}$. Il percorso seguito dall'Impero Ottomano, denotando peculiarità non riscontrabili altrove, rispecchiò quello intrapreso da altre famiglie reali, Romanov e Asburgo ma anche Hannover e Hohenzollern, definito da Anderson come processo di "naturalizzazione" delle dinastie europee, il cui esito principale fu l'emergere dei nazionalismi ufficiali in quanto «reazione ai movimenti nazionali popolari che, sin dal 1821, proliferarono in Europa - nonché - estremo tentativo di nascondere le molte discrepanze tra l’idea di nazione e la realtà dinastica» ${ }^{67}$.

Il pan-islamismo hamidiano, attraverso il rilancio del carattere islamico della società, fu orientato a costruire un vasto consenso interno nel tentativo di salvare l'Impero Ottomano dallo smembramento ${ }^{68}$. Per questo motivo è corretto definirlo un pan-islamismo difensivo distante dai successivi movimenti pan-islamici del XX secolo che invece mirarono e mirano tuttora ad espandere i confini del dār al-Islām. L'idea di compattare i sudditi dell'impero, richiamando la cittadinanza ottomana, base dell'ottomanismo liberale e dei Giovani Ottomani, mostra la continuità con i precedenti progetti ottomani; infatti, il fine ultimo dell'ottomanismo hamidiano non fu la creazione di un'entità sovranazionale ma, per dirla con le parole di Hobsbawm, di una modernizzazione che conservasse l'antico ordinamento della subordinazione sociale ${ }^{69}$.

La scelta di Abdülhamid II si rivelò adatta a dare sfogo alle spinte in chiave islamica provenienti dal basso, espressione del tradizionalismo contadino, e a quelle provenienti dall'intellighenzia islamica influenzata dai Giovani Ottomani. L'invenzione e il recupero di antiche tradizioni aiutarono a giustificare il ruolo autoritario del sovrano e a rafforzarne l'immagine in campo internazionale. Come nel resto d'Europa, le tradizioni inventate diedero sicurezza perché rappresentavano quanto restava di immutabile in un'epoca di fluidità e cambiamento.

Il limite hamidiano fu l'incapacità di suscitare una solidarietà non solo religiosa ma anche identitaria tra i sudditi, aspetto questo che, se realizzato, avrebbe garantito un progetto politico di più ampio respiro. Un limite che indirettamente agevolò il diffondersi degli etno-nazionalismi creando le condizioni per una ulteriore disgregazione del corpo imperiale. Detto questo, i molti elementi di novità introdotti nel contesto ideologico politico ottomano e il loro implicito legame con i movimenti

66 ZÜRCHER, Eric J., «The Ottoman Empire 1850-1922: Unavoidable Failure?», in Leiden University Press Paper, 2006, p. 8.

${ }^{67}$ ANDERSON, Benedict, Imagined Communities: Reflections on the Origin and Spread of Nationalism, London, Verso, 1991, pp. 86-67.

${ }^{68}$ DEL ZANNA, Giorgio, op. cit., pp. 196-198.

${ }^{69}$ HOBSBAWM, Eric J., RANGER, Terence (a cura di), op. cit., p. 256. 
culturali europei, portano a considerare il pan-islamismo hamidiano come un movimento moderno della storia turca segnando da una parte la rottura con le precedenti esperienze "politiche" islamiche ${ }^{70}$, costituendo dall'altra la matrice dei futuri sviluppi politici e identitari turchi (Giovani Turchi e Ataturk). Il patriottismo emerso nel regno hamidiano, tuttavia, si sviluppò in seguito alla rivoluzione costituzionale del 1908, quando un vigoroso sentimento di solidarietà e appartenenza venne incanalato non nella figura del sultano califfo ma nell'idea di nazione, troppo tardi però per resistere all'onda d'urto delle pressioni contingenti.

${ }^{70}$ DERINGIL, Selim, op. cit., p. 67. 


\section{* L'autore}

Federico Donelli dottorando in Scienze Politiche all'Università degli Studi di Genova. I suoi principali interessi di ricerca riguardano le dinamiche socio-politiche interne all'Impero Ottomano durante il XIX secolo, dal sistema coabitativo dei millet agli etno-nazionalismi. Attualmente sta occupandosi della Turchia sotto la guida dell'AKP, in particolare della politica estera impostata da Ahmet Davutoğlu e del recupero dell'eredità ottomana.

URL: < http://www.studistorici.com/progett/autori/\#Donelli >

\section{Per citare questo articolo:}

DONELLI, Federico, «Simboli e tradizioni "inventate" alla corte di Abdülhamid II», Diacronie. Studi di Storia Contemporanea : Le monarchie nell'età dei nazionalismi, 29/12/2013,

URL: < http://www.studistorici.com/2013/12/29/donelli_numero_16/ >

Diacronie Studi di Storia Contemporanea $\beta$ www.diacronie.it

Risorsa digitale indipendente a carattere storiografico. Uscita trimestrale.

redazione.diacronie@hotmail.it

Comitato di redazione: Marco Abram - Jacopo Bassi - Luca Bufarale - Alessandro Cattunar - Elisa Grandi - Deborah Paci - Fausto Pietrancosta - Matteo Tomasoni - Luca Zuccolo

Diritti: gli articoli di Diacronie. Studi di Storia Contemporanea sono pubblicati sotto licenza Creative Commons 2.5. Possono essere riprodotti a patto di non modificarne i contenuti e di non usarli per fini commerciali. La citazione di estratti è comunque sempre autorizzata, nei limiti previsti dalla legge. 